\title{
The coexistence of quasi-periodic and blow-up solutions in a superlinear Duffing equation ${ }^{1}$
}

\author{
Yanmei Sun ${ }^{\mathrm{a}, \mathrm{b}}$, Xiong $\mathrm{Li}^{2 \mathrm{a}}$ \\ ${ }^{a}$ School of Mathematical Sciences, Beijing Normal University, Beijing 100875, P.R. \\ China. \\ ${ }^{b}$ School of Mathematics and Information Sciences, Weifang University, Weifang, \\ Shandong, 261061, P.R. China.
}

\begin{abstract}
In this paper we will construct a continuous positive periodic function $p(t)$ such that the corresponding superlinear Duffing equation

$$
x^{\prime \prime}+a(x) x^{2 n+1}+p(t) x^{2 m+1}=0, \quad n+2 \leq 2 m+1<2 n+1
$$

possesses a solution which escapes to infinity in some finite time, and also has infinitely many subharmonic and quasi-periodic solutions, where the coefficient $a(x)$ is an arbitrary positive smooth periodic function defined in the whole real axis.
\end{abstract}

Keywords: Superlinear Duffing equations; Blow up; Quasi-periodic solutions.

\section{Introduction}

In the early 1960's, Littlewood [8] asked whether all solutions of the second order differential equation

$$
x^{\prime \prime}+V_{x}(x, t)=0
$$

\footnotetext{
${ }^{1}$ Partially supported by the NSFC (11571041) and the Fundamental Research Funds for the Central Universities.

${ }^{2}$ Corresponding author.

URL: sunyanmei2009@126.com (Yanmei Sun), xli@bnu.edu.cn (Xiong Li)
} 
are bounded for all time, that is, $\sup _{t \in \mathbb{R}}\left(|x(t)|+\left|x^{\prime}(t)\right|\right)<+\infty$ holds for all solutions $x(t)$ of Eq.(1.1).

For the Littlewood boundedness problem, during the past years, people have paid more attention to the following equation with the polynomial potentials

$$
x^{\prime \prime}+x^{2 n+1}+\sum_{k=0}^{2 n} p_{k}(t) x^{k}=0,
$$

where $p_{k}(t+1)=p_{k}(t)(k=0,1, \cdots, 2 n)$, since

$$
x^{\prime \prime}+x^{2 n+1}=0
$$

is a very nice time-independent integrable system, of which all solutions are periodic. Thus if $|x|$ is large enough, Eq.(1.2) can be treated as a perturbation of an integrable system, then Moser's twist theorem could be applied to prove the boundedness of all solutions.

The first result was due to Morris [14], who proved that all solutions of the equation with the biquadratic potential

$$
x^{\prime \prime}+2 x^{3}=p(t)=p(t+1)
$$

are bounded.

Using the famous Moser's twist theorem [15], Diekerhoff and Zehnder [1] generalized Morris's results to Eq.(1.2). In [1], the coefficients $p_{k}(t)$ are required to be sufficiently smooth to construct a series of variable changes to transform Eq.(1.2) into a nearly integrable systems for large energies. In fact, in [1], the smoothness on $p_{k}(t)$ depends on the index $k$.

Later, Yuan [20] proved that all solutions of Eq.(1.2) are bounded if $p_{i}(t) \in C^{2}, n+1 \leq i \leq 2 n ; p_{i}(t) \in C^{1}, 0 \leq i \leq n$. Recently, we [5] obtained the same conclusion if $p_{i}(t) \in C^{1}, n+1 \leq i \leq 2 n ; p_{i}(t) \in C^{0}, 0 \leq i \leq n$.

There are other results about the boundedness problem for superlinear Duffing equations during the past years, see [2, 9, 10, 17, 18] and the references therein. As for constructing unbounded solutions for superlinear Duffing equations, there also are some results ([3, 4, 6, 6, 12, 16]). Let us recall the results in [4] and [16]. Levi and You [4] proved that the equation

$$
x^{\prime \prime}+x^{2 n+1}+p(t) x^{2 m+1}=0
$$

with a special discontinuous coefficient $p(t)=K^{[t] \bmod 2}, 0<K<1, n+$ $2 \leq 2 m+1<2 n+1$, possesses an oscillatory unbounded solution. In 
2000, Wang [16] constructed a continuous periodic function $p(t)$ such that the corresponding equation

$$
x^{\prime \prime}+x^{2 n+1}+p(t) x^{i}=0
$$

possesses a solution which escapes to infinity in some finite time, where $n \geq 2$ and $n+2 \leq i<2 n+1$.

In this paper we consider the following second order differential equation

$$
x^{\prime \prime}+a(x) x^{2 n+1}+p(t) x^{2 m+1}=0, \quad n+2 \leq 2 m+1<2 n+1,
$$

where the coefficient $a(x)$ is an arbitrary positive smooth periodic function defined in the whole real axis, will construct a continuous positive periodic function $p(t)$ and obtain the coexistence of quasi-periodic solutions and blowup phenomena for the corresponding equation (1.3). More precisely, we will prove

Theorem 1.1. There exists a continuous positive periodic function $p(t)$ such that the corresponding equation (1.3) possesses a solution which escapes to infinity in some finite time, and also has infinitely many subharmonic and quasi-periodic solutions.

Firstly, we will employ the idea in [16] to construct a continuous positive periodic function $p(t)$ such that the corresponding equation (1.3) possesses a solution which escapes to infinity in some finite time. Here, we will construct the positive periodic function $p(t)$ and the blow up solution $x(t)$ simultaneously.

First of all, we observe that during the time when the curve spirals once around the origin, the action variable $I$ increases at some times and decreases at other times after the action-angle variables $(I, \theta)$ are introduced. Therefore we do not know whether the increment of $I$ is positive or negative. However we can construct a time $t_{1} \ll 1$ and modify $p(t) \equiv 1$ on $[0,1]$ so that the increment of $I$ on this time interval $\left[0, t_{1}\right]$ is positive and equals to $O\left(\frac{1}{\tau} I_{0}^{\frac{2 m-n+2}{n+2}}\right)$ if the initial point $(I(0), \theta(0))=\left(I_{0}, 0\right)$ is far enough from the origin, where the "jump" $\frac{1}{\tau}\left(0<\frac{1}{\tau}<1\right)$ is critical to modify $p(t)$ and to our estimations.

Inductively, we can construct a series of times $t_{1}, t_{2}, \cdots, t_{i}, t_{i+1}, \cdots$ and modify $p(t)$ on $\left[t_{i}, t_{i+1}\right], i=1,2, \cdots$, so that on every such interval $\left[t_{i}, t_{i+1}\right]$, the increment is positive and at least $O\left(\frac{1}{\tau} I_{0}^{\frac{2 m-n+2}{n+2}}\right)$. 
Hence, we can construct a time $T_{1} \leq \frac{1}{\tau^{\prime}}<1$, so that the curve spirals at least $\left[\frac{1}{\tau^{\prime}} \frac{n}{n}^{n+2}\right]$ times around the origin on the interval $\left[0, T_{1}\right]$ and $I_{1}:=$ $I\left(T_{1}\right)>I_{0}+\frac{c}{\tau \tau^{\prime}} I_{0}^{\frac{2 m+2}{n+2}}$ with $c>0$ independent of induction steps, where $\frac{2 m+2}{n+2}>1$ and sufficiently large $\tau^{\prime}$ is used to ensure the blow up time not more than 1 . This complete an induction step: during the interval of time $\left[0, T_{1}\right], I$ increases from $I_{0}$ to $I_{1}$.

Inductively, a series of times $T_{1}, T_{2}, \cdots, T_{i}, T_{i+1}, \cdots$ are constructed such that during the interval of time $\left[T_{k}, T_{k+1}\right], I$ increases from $I_{k}$ to $I_{k+1}$, where $I_{k+1}>I_{k}+\frac{c}{\left(\tau^{\prime}\right)^{k}} I_{k}^{\frac{2 m+2}{n+2}}$ with the jump $\frac{1}{\tau^{k}}$, where $T_{k+1}-T_{k} \leq \frac{1}{\tau^{k} k}$. The reason that the jump is less and less is that we have to assure $p(t)$ is continuous. Because the exponent $\frac{2 m+2}{n+2}>1$, the less and less jump cannot stop the rapid increase of $I$. If $\frac{1}{\tau^{\prime}}$ is chosen small enough, we will find that $T_{k} \rightarrow T_{\infty}<1$ as $k \rightarrow \infty$ and $I(t) \rightarrow+\infty$ as $t \rightarrow T_{\infty}$.

Once we have found the continuous positive periodic function $p(t)$ such that the corresponding equation (1.3) possesses a solution which escapes to infinity in some finite time, the remain thing is to apply the result in [11]. To this end, we first introduce this result. Consider the conservative system

$$
x^{\prime \prime}+p(t) x^{2 m+1}+e(t, x)=0, \quad m \geq 1,
$$

where $p(t)$ is a continuous and 1-periodic function in the time $t, e(t, x)$ is also 1-periodic in the time $t$ and dominated by the power $x^{2 m+2}$ in a neighborhood of $x=0$. Liu in [11] proved that if $\int_{0}^{1} p(t) d t \neq 0$, then the trivial solution $x=0$ of Eq.(1.4) is stable in the Liapunov sense if and only if $\int_{0}^{1} p(t) d t>$ 0 by Moser's twist theorem. Moreover, by the same argument in [1], if $\int_{0}^{1} p(t) d t>0$, then Eq.(1.4) also has infinitely many subharmonic and quasiperiodic solutions with small amplitudes. Compared Eq.(1.3) with Eq.(1.4), since $p(t)$ is positive, then Eq.(1.3) also has infinitely many subharmonic and quasi-periodic solutions with small amplitudes.

Therefore, if we find a continuous positive periodic function $p(t)$ such that the corresponding equation (1.3) possesses a blow-up solution, then such equation also has infinitely many subharmonic and quasi-periodic solutions with small amplitudes simultaneously.

Similar to the above, if we modify $p(t) \equiv 0$ in $[0,1]$, we can construct a continuous non-positive periodic function $p(t)$ with $\int_{0}^{1} p(t) d t<0$ such that the corresponding equation (1.3) possesses a solution which escapes to infinity 
in some finite time, and at the same time, by Liu's result in [11], the trivial solution $x=0$ of such equation is not stable. Therefore we can obtain

Theorem 1.2. There exists a continuous non-positive periodic function $p(t)$ such that the corresponding equation (1.3) possesses a solution which escapes to infinity in some finite time, and the trivial solution $x=0$ is not stable.

Finally we remark that the authors in [19] also obtained the coexistence of quasi-periodic solutions and blow-up phenomena in a class of higher dimensional Duffing-type equations, and the author [13] obtained the coexistence of bounded and unbounded motions in a bouncing ball model.

The paper is organized as follows. The action-angle variables are introduced in Section 2. In Section 3 we first prove some Lemmas which will be useful later. After that, we will construct a continuous positive periodic function $p(t)$ and a series of times $T_{k}$, then obtain an unbounded solution of equation (1.3) and finish the proof of Theorem 1.1 .

\section{Action-angle variables}

In this section we first introduce action and angle variables. Let $y=x^{\prime}$, then Eq.(1.3) is equivalent to the following Hamiltonian system

$$
x^{\prime}=\frac{\partial H}{\partial y}, \quad y^{\prime}=-\frac{\partial H}{\partial x},
$$

where the Hamiltonian is

$$
H(x, y, t)=\frac{1}{2} y^{2}+G(x)+\frac{p(t)}{2 m+2} x^{2 m+2}
$$

with

$$
G(x)=\int_{0}^{x} a(s) s^{2 n+1} d s .
$$

In order to introduce action and angle variables, we consider the auxiliary autonomous system

$$
x^{\prime}=y, \quad y^{\prime}=-G^{\prime}(x) .
$$

Since $a(x)>0$ for all $x \in \mathbb{R}$, then $G(x)>0$ for all $x \neq 0$, and all solutions of this system are periodic. For every $h>0$, denote by $I(h)$ the area enclosed by the closed curve

$$
\Gamma_{h}: \frac{1}{2} y^{2}+G(x)=h .
$$


That is,

$$
I=I(h)=\int_{\Gamma_{h}} \sqrt{2(h-G(x))} d x .
$$

Let $h=h(I)$ be the inverse function of $I=I(h)$. Define

$$
S(x, I)= \begin{cases}\int_{x_{-}}^{x} \sqrt{2(h(I)-G(s))} d s, & y \geq 0, \\ I-\int_{x_{-}}^{x} \sqrt{2(h(I)-G(s))} d s, & y<0,\end{cases}
$$

where $x_{-}=x_{-}(I)<0$ is determined uniquely by $G\left(x_{-}\right)=h(I)$.

Now we introduce the well-known action-angle transformation

$$
y=\frac{\partial S}{\partial x}, \quad \theta=\frac{\partial S}{\partial I} .
$$

Then

$$
\theta= \begin{cases}h^{\prime}(I) \int_{x_{-}}^{x} \frac{d s}{\sqrt{2(h(I)-G(s))}}, & y \geq 0, \\ 1-h^{\prime}(I) \int_{x_{-}}^{x} \frac{d s}{\sqrt{2(h(I)-G(s))}}, & y<0 .\end{cases}
$$

Denote

$$
\Psi:(\theta, I) \rightarrow(x, y),
$$

then under $\Psi$, the Hamiltonian $H$ of (2.1) is transformed into

$$
H_{1}=H \circ \Psi=h(I)+\frac{p(t)}{2 m+2} x(I, \theta)^{2 m+2},
$$

and the corresponding Hamiltonian system is

$$
\left\{\begin{array}{l}
\frac{d \theta}{d t}=h^{\prime}(I)+p(t) x(I, \theta)^{2 m+1} \partial_{I} x(I, \theta), \\
\frac{d I}{d t}=-p(t) x(I, \theta)^{2 m+1} \partial_{\theta} x(I, \theta) .
\end{array}\right.
$$

In the following, we do not attempt to obtain estimates with particularly sharp constants. Indeed, we suppress all constants, and use the notations $u \leq \cdot v$ and $u \cdot \leq v$ to indicate that $u \leq c v$ and $c u \leq v$, respectively, with some constant $c>0$.

Now we give some estimates on $h(I)$ and $x(I, \theta)$. For this purpose, we first give some properties of the potential function $G$. 
Lemma 2.1. For all $x \neq 0$, we have

$$
\begin{gathered}
x^{2 n+2} \cdot \leq G(x) \leq \cdot x^{2 n+2}, \quad\left|G^{\prime}(x)\right| \leq \cdot|x|^{2 n+1} \\
\left|G^{\prime \prime}(x)\right| \leq \cdot\left(|x|^{2 n+1}+x^{2 n}\right), \quad\left|\frac{G(x)}{G^{\prime}(x)}\right| \leq \cdot|x|, \quad\left|\frac{G(x) G^{\prime \prime}(x)}{G^{\prime}(x)^{2}}\right| \leq \cdot(|x|+1) .
\end{gathered}
$$

Proof. Since the periodic function $a(x)$ is positive, then there exist two positive constants $m, M$ such that $0<m \leq a(x) \leq M$ for all $x \in \mathbb{R}$ and from the expression (2.2) of $G(x)$, we know that for $x>0$,

$$
\frac{m}{2 n+2} x^{2 n+2} \leq G(x)=\int_{0}^{x} a(s) s^{2 n+1} d s \leq \frac{M}{2 n+2} x^{2 n+2} ;
$$

and if $x<0$,

$$
\frac{m}{2 n+2} x^{2 n+2} \leq G(x)=\int_{0}^{-x} a(-s) s^{2 n+1} d s \leq \frac{M}{2 n+2} x^{2 n+2},
$$

which yields the first inequality of this lemma.

If $x>0$, then $m x^{2 n+1} \leq G^{\prime}(x)=a(x) x^{2 n+1} \leq M x^{2 n+1}$; if $x<0$, then $M x^{2 n+1} \leq G^{\prime}(x)=a(x) x^{2 n+1} \leq m x^{2 n+1}$. Combining this two inequalities, one can obtain the second inequality.

Since $G^{\prime \prime}(x)=a^{\prime}(x) x^{2 n+1}+(2 n+1) a(x) x^{2 n}$, if we let $m_{1} \leq a^{\prime}(x) \leq M_{1}$ for all $x \in \mathbb{R}$, where $m_{1}<0, M_{1}>0$ are two constants, then $m_{1} x^{2 n+1}+$ $(2 n+1) m x^{2 n} \leq G^{\prime \prime}(x) \leq M_{1} x^{2 n+1}+(2 n+1) M x^{2 n}$ for $x>0$, and $M_{1} x^{2 n+1}+$ $(2 n+1) m x^{2 n} \leq G^{\prime \prime}(x) \leq m_{1} x^{2 n+1}+(2 n+1) M x^{2 n}$ for $x<0$, therefore $\left|G^{\prime \prime}(x)\right| \leq \max \left\{\left|m_{1}\right|, M_{1}\right\}|x|^{2 n+1}+(2 n+1) M x^{2 n} \leq \cdot\left(|x|^{2 n+1}+x^{2 n}\right)$, which is the third inequality.

For $x>0, \frac{m}{M} x \leq \frac{G(x)}{G^{\prime}(x)} \leq \frac{M}{m} x$, and if $x<0, \frac{M}{m} x \leq \frac{G(x)}{G^{\prime}(x)} \leq \frac{m}{M} x$, then the fourth inequality holds.

If $x>0, \frac{m m_{1}}{(2 n+2) M^{2}} x+\frac{(2 n+1) m^{2}}{(2 n+2) M^{2}} \leq \frac{G(x) G^{\prime \prime}(x)}{G^{\prime}(x)^{2}} \leq \frac{M M_{1}}{(2 n+2) m^{2}} x+\frac{(2 n+1) M^{2}}{(2 n+2) m^{2}}$, and if $x<0, \frac{m M_{1}}{(2 n+2) M^{2}} x+\frac{(2 n+1) m^{2}}{(2 n+2) M^{2}} \leq \frac{G(x) G^{\prime \prime}(x)}{G^{\prime}(x)^{2}} \leq \frac{M m_{1}}{(2 n+2) m^{2}} x+\frac{(2 n+1) M^{2}}{(2 n+2) m^{2}}$, hence

$$
\left|\frac{G(x) G^{\prime \prime}(x)}{G^{\prime}(x)^{2}}\right| \leq \cdot(|x|+1) .
$$

Up to now, we have finished the proof of the lemma. 
Lemma 2.2. For sufficiently large $h>0$, we have

$$
\begin{gathered}
h^{\frac{1}{2}+\frac{1}{2 n+2}} \cdot \leq I(h) \leq \cdot h^{\frac{1}{2}+\frac{1}{2 n+2}}, \\
h^{-\frac{1}{2}+\frac{1}{2 n+2}} \cdot \leq I^{\prime}(h) \leq \cdot h^{-\frac{1}{2}+\frac{1}{2 n+2}} \\
\left|I^{\prime \prime}(h)\right| \leq \cdot h^{-\frac{3}{2}+\frac{1}{n+1}} .
\end{gathered}
$$

Proof. Let $x_{-}<0<x_{+}$defined by $G\left(x_{-}\right)=G\left(x_{+}\right)=h$ for any $h>0$, then

$$
I(h)=2 \sqrt{2} \int_{x_{-}}^{x_{+}} \sqrt{h-G(x)} d x .
$$

By Lemma 2.1, we know that

$$
h^{\frac{1}{2 n+2}} \cdot \leq x_{+}(h),\left|x_{-}(h)\right| \leq \cdot h^{\frac{1}{2 n+2}},
$$

which implies that

$$
I(h) \leq \cdot h^{\frac{1}{2}+\frac{1}{2 n+2}} .
$$

On the other hand, if we let $\bar{x}>0$ determined by $G(\bar{x})=\frac{h}{2}$, then

$$
h^{\frac{1}{2 n+2}} \cdot \leq \bar{x}(h) \leq \cdot h^{\frac{1}{2 n+2}}
$$

and for $0 \leq s \leq \bar{x}$

$$
\sqrt{h-G(s)} \geq \sqrt{h-G(\bar{x})} \geq \cdot h^{\frac{1}{2}}
$$

thus

$$
I(h) \geq 2 \sqrt{2} \int_{0}^{\bar{x}} \sqrt{h-G(s)} d s \geq \cdot h^{\frac{1}{2}+\frac{1}{2 n+2}} .
$$

Now we prove the second inequality. From the expression of $I(h)$, we know that

$$
I^{\prime}(h)=\sqrt{2} \int_{x_{-}}^{x_{+}} \frac{d x}{\sqrt{h-G(x)}}=\sqrt{2} \int_{x_{-}}^{0} \frac{d x}{\sqrt{h-G(x)}}+\sqrt{2} \int_{0}^{x_{+}} \frac{d x}{\sqrt{h-G(x)}} .
$$

The second term can be rewritten as follows

$$
\int_{0}^{x_{+}} \frac{d x}{\sqrt{h-G(x)}}=\int_{0}^{\bar{x}} \frac{d x}{\sqrt{h-G(x)}}+\int_{\bar{x}}^{x_{+}} \frac{d x}{\sqrt{h-G(x)}} .
$$


For $0 \leq x \leq \bar{x}$, we have

$$
h^{-\frac{1}{2}} \cdot \leq \frac{1}{\sqrt{h-G(x)}} \leq \cdot h^{-\frac{1}{2}},
$$

which together with (2.4) implies that

$$
h^{-\frac{1}{2}+\frac{1}{2 n+2}} \cdot \leq \int_{0}^{\bar{x}} \frac{d x}{\sqrt{h-G(x)}} \leq \cdot h^{-\frac{1}{2}+\frac{1}{2 n+2}} .
$$

If $\bar{x} \leq x \leq x_{+}$, then

$$
h-G(x)=G\left(x_{+}\right)-G(x)=G^{\prime}(\xi)\left(x_{+}-x\right), \quad \xi \in\left(x, x_{+}\right) \subset\left(\bar{x}, x_{+}\right)
$$

and

$$
h^{1-\frac{1}{2 n+2}} \cdot \leq \bar{x}^{2 n+1} \cdot \leq G^{\prime}(\xi)=a(\xi) \xi^{2 n+1} \leq \cdot x_{+}^{2 n+1} \leq \cdot h^{1-\frac{1}{2 n+2}}
$$

which implies that

$$
\frac{h^{-\frac{1}{2}+\frac{1}{4 n+4}}}{\sqrt{x_{+}-x}} \cdot \leq \frac{1}{\sqrt{h-G(x)}} \leq \cdot \frac{h^{-\frac{1}{2}+\frac{1}{4 n+4}}}{\sqrt{x_{+}-x}} .
$$

Combining the last equation with the fact that

$$
h^{\frac{1}{4 n+4}} \cdot \leq \int_{\bar{x}}^{x_{+}} \frac{d x}{\sqrt{x_{+}-x}} \leq \cdot h^{\frac{1}{4 n+4}},
$$

we obtain

$$
h^{-\frac{1}{2}+\frac{1}{2 n+2}} \cdot \leq \int_{\bar{x}}^{x_{+}} \frac{d x}{\sqrt{h-G(x)}} \leq \cdot h^{-\frac{1}{2}+\frac{1}{2 n+2}}
$$

and thus

$$
h^{-\frac{1}{2}+\frac{1}{2 n+2}} \cdot \leq \int_{0}^{x_{+}} \frac{d x}{\sqrt{h-G(x)}} \leq \cdot h^{-\frac{1}{2}+\frac{1}{2 n+2}} .
$$

Similarly, one can prove that

$$
h^{-\frac{1}{2}+\frac{1}{2 n+2}} \cdot \leq \int_{x_{-}}^{0} \frac{d x}{\sqrt{h-G(x)}} \leq \cdot h^{-\frac{1}{2}+\frac{1}{2 n+2}},
$$

which completes the proof of the second inequality. 
Finally we prove the estimate on $I^{\prime \prime}(h)$. From [2], we know that

$$
I^{\prime \prime}(h)=\frac{\sqrt{2}}{h} \int_{x_{-}}^{x_{+}}\left(\frac{1}{2}-\frac{G(x) G^{\prime \prime}(x)}{G^{\prime}(x)^{2}}\right) \frac{d x}{\sqrt{h-G(x)}} .
$$

By Lemma 2.1, for sufficiently large $h>0$, we have

$$
\left|\frac{G(x) G^{\prime \prime}(x)}{G^{\prime}(x)^{2}}\right| \leq \cdot(|x|+1) \leq \cdot h^{\frac{1}{2 n+2}}
$$

and thus

$$
\left|I^{\prime \prime}(h)\right| \leq \cdot h^{\frac{1}{2 n+2}-1} I^{\prime}(h) \leq \cdot h^{-\frac{3}{2}+\frac{1}{n+1}},
$$

which finishes the proof of this lemma.

Since $h=h(I)$ is the inverse function of $I=I(h)$, we immediately obtain

Lemma 2.3. For sufficiently large $I>0$, we have

$$
I^{\frac{2 n+2}{n+2}} \cdot \leq h(I) \leq \cdot I^{\frac{2 n+2}{n+2}}, \quad I^{\frac{n}{n+2}} \cdot \leq h^{\prime}(I) \leq \cdot I^{\frac{n}{n+2}}, \quad\left|h^{\prime \prime}(I)\right| \leq \cdot I^{-\frac{1}{n+2}} .
$$

Now we give some estimates on $x(I, \theta)$.

Lemma 2.4. For sufficiently large $I>0$, we have

$$
\begin{gathered}
\partial_{\theta} x>0 \text {, when } y>0, \quad \partial_{\theta} x<0 \text {, when } y<0 ; \\
|x| \leq \cdot I^{\frac{1}{n+2}}, \quad\left|\partial_{\theta} x\right| \leq \cdot I^{\frac{1}{n+2}}, \quad\left|\partial_{I} x\right| \leq \cdot I^{-\frac{n}{n+2} .}
\end{gathered}
$$

Proof. The inequality $|x| \leq \cdot I^{\frac{1}{n+2}}$ is obvious. According to the definition of $\theta$, when $y>0\left(\right.$ or $0<\theta<\frac{1}{2}$ ), we have

$$
\theta=h^{\prime}(I) \int_{x_{-}}^{x} \frac{d s}{\sqrt{2(h(I)-G(s))}} .
$$

Differentiating the above equality with respect to $\theta$ yields that

$$
1=\frac{h^{\prime}(I)}{\sqrt{2(h(I)-G(x))}} \partial_{\theta} x
$$

which implies that

$$
\partial_{\theta} x=\frac{\sqrt{2(h(I)-G(x))}}{h^{\prime}(I)} .
$$


Therefore, $\partial_{\theta} x>0$ for $0<\theta<\frac{1}{2}$, and by Lemma 2.3, $\partial_{\theta} x \leq \cdot I^{\frac{1}{n+2}}$ holds.

Similarly, when $y<0$ (or $\frac{1}{2}<\theta<1$ ), we have

$$
\partial_{\theta} x=-\frac{\sqrt{2(h(I)-G(x))}}{h^{\prime}(I)},
$$

hence $\partial_{\theta} x<0$ and $\left|\partial_{\theta} x\right| \leq \cdot I^{\frac{1}{n+2}}$.

Now we prove the estimate on $\partial_{I} x$. From [2], when $y \geq 0\left(\right.$ or $\left.0 \leq \theta \leq \frac{1}{2}\right)$, we have

$$
\partial_{I} x=\sqrt{2(h(I)-G(x))} \int_{x_{-}}^{x} \frac{L(I, s) d s}{\sqrt{2(h(I)-G(s))}}+\frac{h^{\prime}(I)}{h(I)} \frac{G(x)}{G^{\prime}(x)},
$$

where

$$
L(I, x)=-\frac{h^{\prime \prime}(I)}{h^{\prime}(I)}-\frac{h^{\prime}(I)}{2 h(I)}\left(1-\frac{2 G(x) G^{\prime \prime}(x)}{G^{\prime}(x)^{2}}\right) .
$$

According to Lemmas 2.1, 2.3, $|L| \leq \cdot I^{-\frac{n+1}{n+2}}$ and $\left|\frac{h^{\prime}(I)}{h(I)} \frac{G(x)}{G^{\prime}(x)}\right| \leq \cdot I^{-\frac{n+1}{n+2}}$ hold. Also, since for $x_{-} \leq s \leq x, \frac{\sqrt{2(h(I)-G(x))}}{\sqrt{2(h(I)-G(s))}} \leq 1$, then $\left|\partial_{I} x\right| \leq \cdot I^{-\frac{n}{n+2}}$. One can obtain the same estimate for $y<0$. Thus, we have finished the proof of this lemma.

If we define $x_{1}, x_{2}, x_{3}$ by $x(I, \theta)=I^{\frac{1}{n+2}} x_{1}(I, \theta), \quad \partial_{I} x(I, \theta)=I^{-\frac{n}{n+2}} x_{2}(I, \theta), \quad \partial_{\theta} x(I, \theta)=I^{\frac{1}{n+2}} x_{3}(I, \theta)$, then they are bounded functions for sufficiently large $I$ and all $\theta \in \mathbb{R}$, that is, there are three positive constants $B_{1}, B_{2}, B_{3}$ such that

$$
\left|x_{i}(I, \theta)\right| \leq B_{i}, \quad i=1,2,3 .
$$

Furthermore, there exist two positive constants $C_{1}, C_{2}$ such that

$$
-x_{1}(I, \theta) \geq C_{1}, \quad x_{3}(I, \theta) \geq C_{2}
$$

for sufficiently large $I$ and $\theta \in\left[\frac{1}{16}, \frac{3}{16}\right]$. Moreover, we can rewrite system (2.3) into

$$
\left\{\begin{array}{l}
\frac{d \theta}{d t}=h^{\prime}(I)+p(t) I^{\frac{2 m+1-n}{n+2}} x_{1}(I, \theta)^{2 m+1} x_{2}(I, \theta), \\
\frac{d I}{d t}=-p(t) I^{\frac{2 m+2}{n+2}} x_{1}(I, \theta)^{2 m+1} x_{3}(I, \theta) .
\end{array}\right.
$$

By Lemma 2.3, we know that $I^{\frac{n}{n+2}} \cdot \leq h^{\prime}(I) \leq \cdot I^{\frac{n}{n+2}}$. Also by our assumption, $2 m+1 \leq 2(n-1)+1$, thus $2 m+1-n \leq n-1$ and the right of the first equation in (2.7) is dominated by $h^{\prime}(I)$ for sufficiently large $I$. 


\section{The proof of Theorem 1.1}

Now we define $p(t)$ in $[0,1]$. We will construct a time $t_{1}<1$ and modify $p^{0}(t) \equiv 1$ on $[0,1]$ so that the action of one solution of (2.7) increases in $\left[0, t_{1}\right]$. We divide the construction into two steps: first, we construct a piecewise continuous function $p^{1}(t)$ so that the action of one solution of (2.7) obtains a positive increment in $\left[0, t_{1}\right]$ as we expect. Then we modify this function $p^{1}(t)$ into a continuous one in such a way that the modification does not influence the estimate we had obtained before.

Without loss of generality, we assume that the function $a(x)$ is even. Denote the corresponding Hamiltonian system (2.7) with the coefficient function $p(t)$ by $X_{p}$. Suppose the solution $(I(t), \theta(t))$ of $X_{p^{0}}$ with $(I(0), \theta(0))=\left(I_{0}, 0\right)$ at $t=0$ arrives at $\left(I_{\frac{1}{4}}, \frac{1}{4}\right)$ at $t=t_{\frac{1}{4}} \ll 1$, where $I_{0}$ is a sufficiently large constant which will be determined later. Define $p^{\frac{1}{4}}(t)$ be a piecewise continuous function as follows

$$
p^{\frac{1}{4}}(t)= \begin{cases}1, & t \in\left[0, t_{\frac{1}{4}}\right], \\ 1-\sigma, & t \in\left(t_{\frac{1}{4}}, 1\right]\end{cases}
$$

where $0<\sigma<1$ is the jump, which is used to control the increment of $I$.

Suppose the solution $(I(t), \theta(t))$ of $X_{p^{\frac{1}{4}}}$ with $(I(0), \theta(0))=\left(I_{0}, 0\right)$ at $t=0$ arrives at $\left(I_{\frac{1}{2}}, \frac{1}{2}\right)$ at $t=t_{\frac{1}{2}} \ll 1$. Define $p^{\frac{1}{2}}(t)$ be a piecewise continuous function as follows

$$
p^{\frac{1}{2}}(t)= \begin{cases}1, & t \in\left[0, t_{\frac{1}{4}}\right] \\ 1-\sigma, & t \in\left(t_{\frac{1}{4}}, t_{\frac{1}{2}}\right], \\ 1, & t \in\left(t_{\frac{1}{2}}, 1\right] .\end{cases}
$$

Suppose the solution $(I(t), \theta(t))$ of $X_{p^{\frac{1}{2}}}$ with $(I(0), \theta(0))=\left(I_{0}, 0\right)$ at $t=0$ arrives at $\left(I_{\frac{3}{4}}, \frac{3}{4}\right)$ at $t=t_{\frac{3}{4}} \ll 1$. Define $p^{\frac{3}{4}}(t)$ be a piecewise continuous function as follows

$$
p^{\frac{3}{4}}(t)= \begin{cases}1, & t \in\left[0, t_{\frac{1}{4}}\right] \\ 1-\sigma, & t \in\left(t_{\frac{1}{4}}, t_{\frac{1}{2}}\right] \\ 1, & t \in\left(t_{\frac{1}{2}}, t_{\frac{3}{4}}\right] \\ 1, & t \in\left(t_{\frac{3}{4}}, 1\right] .\end{cases}
$$


Suppose the solution $(I(t), \theta(t))$ of $X_{p^{\frac{3}{4}}}$ with $(I(0), \theta(0))=\left(I_{0}, 0\right)$ at $t=0$ arrives at $\left(I_{1}, 1\right)$ at $t=t_{1} \ll 1$. Define $p^{1}(t)$ be a piecewise continuous function as follows

$$
p^{1}(t)= \begin{cases}1, & t \in\left[0, t_{\frac{1}{4}}\right], \\ 1-\sigma, & t \in\left(t_{\frac{1}{4}}, t_{\frac{1}{2}}\right] \\ 1, & t \in\left(t_{\frac{1}{2}}, t_{\frac{3}{4}}\right], \\ 1-\sigma, & t \in\left(t_{\frac{3}{4}}, t_{1}\right], \\ 1, & t \in\left(t_{1}, 1\right] .\end{cases}
$$

That is to say, the solution $(I(t), \theta(t))$ of $X_{p^{1}}$ with $(I(0), \theta(0))=\left(I_{0}, 0\right)$ at $t=0$ arrives at $\left(I_{\frac{1}{4}}, \frac{1}{4}\right)$ at $t=t_{\frac{1}{4}}$, arrives at $\left(I_{\frac{1}{2}}, \frac{1}{2}\right)$ at $t=t_{\frac{1}{2}}$, arrives at $\left(I_{\frac{3}{4}}, \frac{3}{4}\right)$ at $t=t_{\frac{3}{4}}$, arrives at $\left(I_{1}, 1\right)$ at $t=t_{1}$, which finishes one cycle of the construction of $p(t)$.

Now we estimate the differences $I_{1}-I_{0}$ and $t_{1}-t_{0}$.

Lemma 3.1. If $I_{0}$ is sufficiently large, then

$$
\begin{gathered}
I_{0}^{-\frac{n}{n+2}} \cdot \leq t_{\frac{1}{4}} \leq \cdot I_{0}^{-\frac{n}{n+2}}, \\
I_{0}^{\frac{2 m+2-n}{n+2}} \cdot \leq I_{\frac{1}{4}}-I_{0} \leq \cdot I_{0}^{\frac{2 m+2-n}{n+2}} .
\end{gathered}
$$

Proof. Because $x_{1}<0, x_{3}>0$ for $\theta \in\left(0, \frac{1}{4}\right)$, then $\frac{d I}{d t}>0$ for $t \in\left(0, t_{\frac{1}{4}}\right)$ and thus $I(t)$ is an increasing function in this interval. Integrating the first equation of (2.7) from $t=0$ to $t=t_{\frac{1}{4}}$ yields that

$$
t_{\frac{1}{4}} \leq \frac{1}{4}\left(h^{\prime}\left(I_{0}\right)-B_{1}^{2 m+1} B_{2} I_{0}^{\frac{2 m+1-n}{n+2}}\right)^{-1} \leq \cdot I_{0}^{-\frac{n}{n+2}}
$$

for sufficiently large $I_{0}>0$, here we use the estimate on $h^{\prime}(I)$ in Lemma 2.3 and the bound $B_{i}$ of $x_{i}$ in (2.5).

From the second equation of (2.7), we have

$$
\frac{d I}{I^{\frac{2 m+2}{n+2}}}=-x_{1}(I, \theta)^{2 m+1} x_{3}(I, \theta) d t .
$$


Since $x_{1}<0, x_{3}>0$ for $\theta \in\left(0, \frac{1}{4}\right)$, and also integrating the above equation (3.3) from $t=0$ to $t=t_{\frac{1}{4}}$, one can obtain

$$
\frac{n+2}{2 m-n}\left(I_{\frac{1}{4}}^{-\frac{2 m-n}{n+2}}-I_{0}^{-\frac{2 m-n}{n+2}}\right)=\int_{0}^{t^{\frac{1}{4}}} x_{1}(I, \theta)^{2 m+1} x_{3}(I, \theta) d t,
$$

and by (3.2), we get

$$
\frac{n+2}{2 m-n}\left(I_{0}^{-\frac{2 m-n}{n+2}}-I_{\frac{1}{4}}^{-\frac{2 m-n}{n+2}}\right) \leq c B_{1}^{2 m+1} B_{2} I_{0}^{-\frac{n}{n+2}}
$$

where the constant $c>0$ is given by (3.2), which implies that

$$
I_{\frac{1}{4}}^{-\frac{2 m-n}{n+2}} \geq I_{0}^{-\frac{2 m-n}{n+2}}-\tilde{c} I_{0}^{-\frac{n}{n+2}}=I_{0}^{-\frac{2 m-n}{n+2}}\left(1-\tilde{c} I_{0}^{-\frac{2 n-2 m}{n+2}}\right),
$$

here the constant $\tilde{c}=c \frac{2 m-n}{n+2} B_{1}^{2 m+1} B_{2}>0$. Hence we obtain

$$
I_{\frac{1}{4}} \leq I_{0}\left(1-\tilde{c} I_{0}^{-\frac{2 n-2 m}{n+2}}\right)^{-\frac{n+2}{2 m-n}}
$$

which leads to

$$
I_{\frac{1}{4}}-I_{0} \leq \cdot I_{0}^{\frac{2 m+2-n}{n+2}}
$$

On the other hand, from the first equation of (2.7), we have

$$
\begin{aligned}
t_{\frac{1}{4}} & \geq \frac{1}{4}\left(h^{\prime}\left(I_{\frac{1}{4}}\right)+B_{1}^{2 m+1} B_{2} I_{\frac{1}{4}}^{\frac{2 m+1-n}{n+2}}\right)^{-1} \\
& \geq \cdot\left(I_{\frac{1}{4}}^{\frac{n}{n+2}}+I_{\frac{1}{4}}^{\frac{2 m+1-n}{n+2}}\right)^{-1} \\
& \geq \cdot I_{\frac{1}{4}}^{-\frac{n}{n+2}} \\
& \geq \cdot I_{0}^{-\frac{n}{n+2}} .
\end{aligned}
$$

Finally, it follows from (2.6) and (3.4) that

$$
\frac{n+2}{2 m-n}\left(I_{0}^{-\frac{2 m-n}{n+2}}-I_{\frac{1}{4}}^{-\frac{2 m-n}{n+2}}\right) \geq c C_{1}^{2 m+1} C_{2}\left(t_{\frac{3}{16}}-t_{\frac{1}{16}}\right) .
$$


Similarly, the following estimate

$$
t_{\frac{3}{16}}-t_{\frac{1}{16}} \geq \cdot I_{0}^{-\frac{n}{n+2}}
$$

holds. Combining the two inequalities above yields that

$$
I_{0}^{-\frac{2 m-n}{n+2}}-I_{\frac{1}{4}}^{-\frac{2 m-n}{n+2}} \geq \cdot I_{0}^{-\frac{n}{n+2}}
$$

and

$$
I_{\frac{1}{4}}-I_{0} \geq \cdot I_{0}^{\frac{2 m+2-n}{n+2}}
$$

Lemma 3.2. If $I_{0}$ is sufficiently large, then

$$
\begin{gathered}
I_{0}^{-\frac{n}{n+2}} \cdot \leq t_{\frac{1}{2}}-t_{\frac{1}{4}} \leq \cdot I_{0}^{-\frac{n}{n+2}} \\
(1-\sigma) I_{0}^{\frac{2 m+2-n}{n+2}} \cdot \leq I_{\frac{1}{4}}-I_{\frac{1}{2}} \leq \cdot(1-\sigma) I_{0}^{\frac{2 m+2-n}{n+2}} .
\end{gathered}
$$

Proof. Because $x_{1}>0, x_{3}>0$ for $\theta \in\left(\frac{1}{4}, \frac{1}{2}\right)$, then $\frac{d I}{d t}<0$ for $t \in\left(t_{\frac{1}{4}}, t_{\frac{1}{2}}\right)$ and thus $I(t)$ is an decreasing function in this interval. Integrating the first equation of (2.7) from $t=t_{\frac{1}{4}}$ to $t=t_{\frac{1}{2}}$ yields that

$$
I_{\frac{1}{4}}^{-\frac{n}{n+2}} \cdot \leq t_{\frac{1}{2}}-t_{\frac{1}{4}} \leq \cdot I_{\frac{1}{2}}^{-\frac{n}{n+2}}
$$

From the second equation of (2.7), we have

$$
(1-\sigma) I_{\frac{1}{4}}^{-\frac{n}{n+2}} \cdot \leq I_{\frac{1}{2}}^{-\frac{2 m-n}{n+2}}-I_{\frac{1}{4}}^{-\frac{2 m-n}{n+2}} \leq \cdot(1-\sigma) I_{\frac{1}{2}}^{-\frac{n}{n+2}},
$$

and thus

$$
(1-\sigma) I_{\frac{1}{4}}^{\frac{2 m+2-n}{n+2}} \cdot \leq I_{\frac{1}{4}}-I_{\frac{1}{2}} \leq \cdot(1-\sigma) I_{\frac{1}{2}}^{\frac{2 m+2-n}{n+2}} .
$$

By Lemma 3.1, we have

$$
I_{\frac{1}{4}} \leq I_{0}\left(1+c I_{0}^{\frac{2 m-2 n}{n+2}}\right)
$$

with some constant $c>0$, which implies that

$$
I_{\frac{1}{4}}^{-\frac{n}{n+2}} \geq \cdot I_{0}^{-\frac{n}{n+2}}
$$


holds for sufficiently large $I_{0}>0$. Meanwhile it follows from (3.6) that

$$
I_{\frac{1}{2}} \geq \cdot I_{\frac{1}{4}},
$$

which together with $I_{\frac{1}{4}}>I_{0}$ implies that

$$
I_{\frac{1}{2}} \geq \cdot I_{0},
$$

and

$$
I_{\frac{1}{2}}^{-\frac{n}{n+2}} \leq \cdot I_{0}^{-\frac{n}{n+2}} .
$$

Combining (3.5), (3.7) with (3.9), we obtain

$$
I_{0}^{-\frac{n}{n+2}} \cdot \leq t_{\frac{1}{2}}-t_{\frac{1}{4}} \leq \cdot I_{0}^{-\frac{n}{n+2}} .
$$

Also, according to (3.6), (3.8) and $I_{\frac{1}{4}} \geq I_{0}$, one can obtain the second inequality in this lemma.

Using the same method, one can prove the following result.

Lemma 3.3. If $I_{0}$ is sufficiently large, then

$$
\begin{gathered}
I_{0}^{-\frac{n}{n+2}} \cdot \leq t_{\frac{3}{4}}-t_{\frac{1}{2}} \leq \cdot I_{0}^{-\frac{n}{n+2}}, \\
I_{0}^{-\frac{n}{n+2}} \cdot \leq t_{1}-t_{\frac{3}{4}} \leq \cdot I_{0}^{-\frac{n}{n+2}}, \\
I_{0}^{\frac{2 m+2-n}{n+2}} \cdot \leq I_{\frac{3}{4}}-I_{\frac{1}{2}} \leq \cdot I_{0}^{\frac{2 m+2-n}{n+2}}, \\
(1-\sigma) I_{0}^{\frac{2 m+2-n}{n+2}} \cdot \leq I_{\frac{3}{4}}-I_{1} \leq \cdot(1-\sigma) I_{0}^{\frac{2 m+2-n}{n+2}} .
\end{gathered}
$$

Combining Lemmas 3.1, 3.2 and 3.3, we can obtain immediately the estimates on the time $t_{1}$ when the curve spirals once around the origin and the increment of the action variable $I_{1}-I_{0}$.

Lemma 3.4. If $I_{0}$ is sufficiently large, then

$$
\begin{gathered}
I_{0}^{-\frac{n}{n+2}} \cdot \leq t_{1} \leq \cdot I_{0}^{-\frac{n}{n+2}}, \\
\sigma I_{0}^{\frac{2 m+2-n}{n+2}} \cdot \leq I_{1}-I_{0} \leq \sigma I_{0}^{\frac{2 m+2-n}{n+2}} .
\end{gathered}
$$


Now we modify the piecewise continuous function $p^{1}(t)$ of (3.1) into a continuous one. Being short of signs, we keep the notations unchanged in the process of modification. For example, $p^{1}(t)$ denotes the continuous function modified from the original piecewise continuous function $p^{1}(t)$.

First we modify $p^{1}(t)$ on the interval $\left[t_{\frac{1}{4}}, t_{\frac{1}{4}}+I_{0}^{-\eta}\right]\left(\eta>\frac{n}{n+2}\right)$ to be $\sigma\left(t_{\frac{1}{4}}-\right.$ t) $I_{0}^{\eta}+1$. It is easy to see that $\left\{\left(t, p^{1}(t)\right): t \in\left[t_{\frac{1}{4}}, t_{\frac{1}{4}}+I_{0}^{-\eta}\right]\right\}$ is the line segment connecting $\left(t_{\frac{1}{4}}, 1\right)$ and $\left(t_{\frac{1}{4}}+I_{0}^{-\eta}, 1-\sigma\right)$.

In view of the mean value theorem, there must exist a unique new time $t_{\frac{1}{2}}$ such that $\theta\left(t_{\frac{1}{2}}\right)=\frac{1}{2}$ if we let

$$
p^{\frac{1}{2}}(t)= \begin{cases}1, & t \in\left[0, t_{\frac{1}{4}}\right], \\ \sigma\left(t_{\frac{1}{4}}-t\right) I_{0}^{\eta}+1, & t \in\left(t_{\frac{1}{4}}, t_{\frac{1}{4}}+I_{0}^{-\eta}\right], \\ 1-\sigma, & t \in\left(t_{\frac{1}{4}}+I_{0}^{-\eta}, t_{\frac{1}{2}}-I_{0}^{-\eta}\right], \\ \sigma\left(t-t_{\frac{1}{2}}\right) I_{0}^{\eta}+1, & t \in\left(t_{\frac{1}{2}}-I_{0}^{-\eta}, t_{\frac{1}{2}}\right], \\ 1, & t \in\left(t_{\frac{1}{2}}, 1\right] .\end{cases}
$$

Similarly, there exist the unique $t_{\frac{3}{4}}$ and $t_{1}$ such that $\theta\left(t_{\frac{3}{4}}\right)=\frac{3}{4}$ and $\theta\left(t_{1}\right)=$ 1 for $X_{p^{1}}$ with

$$
p^{1}(t)= \begin{cases}1, & t \in\left[0, t_{\frac{1}{4}}\right], \\ \sigma\left(t_{\frac{1}{4}}-t\right) I_{0}^{\eta}+1, & t \in\left(t_{\frac{1}{4}}, t_{\frac{1}{4}}+I_{0}^{-\eta}\right], \\ 1-\sigma, & t \in\left(t_{\frac{1}{4}}+I_{0}^{-\eta}, t_{\frac{1}{2}}-I_{0}^{-\eta}\right], \\ \sigma\left(t-t_{\frac{1}{2}}\right) I_{0}^{\eta}+1, & t \in\left(t_{\frac{1}{2}}-I_{0}^{-\eta}, t_{\frac{1}{2}}\right], \\ 1, & t \in\left(t_{\frac{1}{2}}, t_{\frac{3}{4}}\right], \\ \sigma\left(t_{\frac{3}{4}}-t\right) I_{0}^{\eta}+1, & t \in\left(t_{\frac{3}{4}}, t_{\frac{3}{4}}+I_{0}^{-\eta}\right], \\ 1-\sigma, & t \in\left(t_{\frac{3}{4}}+I_{0}^{-\eta}, t_{1}-I_{0}^{-\eta}\right], \\ \sigma\left(t-t_{1}\right) I_{0}^{\eta}+1, & t \in\left(t_{1}-I_{0}^{-\eta}, t_{1}\right], \\ 1, & t \in\left(t_{1}, 1\right] .\end{cases}
$$

Now the newest coefficient is already a continuous function. It is easy to check that Lemmas 3.1 3.4 still hold with different constants after this modification in view of $I_{0}^{-\eta} \ll I_{0}^{-\frac{n}{n+2}}$. 
We will modify $p^{0}$ inductively and denote the function obtained and the corresponding solution with $\left(I_{0}, 0\right)$ as the initial point by $p^{i}$ and $\left(I^{i}(t), \theta^{i}(t)\right)$ with $\left(I^{i}\left(t_{i}\right), \theta^{i}\left(t_{i}\right)\right)=\left(I_{i}, i\right)$, respectively.

Suppose that we have obtained $p^{0}, p^{1}, \cdots, p^{i}$. The function $p^{i+1}$ defined on $[0,1]$ is constructed by modifying $p^{i}$ on the interval $\left[t_{i}, t_{i+1}\right]$, where $t_{i+1}$ satisfies $\theta^{i+1}\left(t_{i+1}\right)=i+1$ in the same way as above if we regard $I_{i}, t_{i}$ as $I_{0}, t_{0}$. All the lemmas are true after the modification.

In the process of constructing $p^{i}$, we keep the jump $\sigma=\frac{1}{\tau}(\tau \geq 2)$ unchanged until $i=j_{1}$. Then we let $\sigma=\frac{1}{\tau^{2}}$ and keep it unchanged until $i=j_{2}$. Inductively, we choose $\sigma=\frac{1}{\tau^{k}}$ when $\theta \in\left[j_{k-1}, j_{k}\right]$, where $j_{0}=0, j_{1}, j_{2}, \cdots$ are defined as below.

Let $j_{1}=\left[\frac{1}{\tau^{\prime}} I_{0}^{\frac{n}{n+2}}\right]$, where $[x]$ denotes the integer part of $x$ and $\tau^{\prime}>0$ is used to control time and will be determined later. It follows that

$$
T_{1}:=t_{j_{1}} \leq j_{1} \cdot t_{1} \cdot \leq \frac{1}{\tau^{\prime}} I_{0}^{\frac{n}{n+2}} \cdot I_{0}^{-\frac{n}{n+2}} \cdot \leq \frac{1}{\tau^{\prime}} .
$$

On the interval $\left[0, T_{1}\right]$, since $I_{k+1}-I_{k} \geq \cdot \frac{1}{\tau} I_{0}^{\frac{2 m+2-n}{n+2}}$ for $k=0,1, \cdots, j_{1}-1$, we have

$$
I_{j_{1}}:=I^{j_{1}}\left(T_{1}\right) \geq \cdot\left[\frac{1}{\tau^{\prime}} I_{0}^{\frac{n}{n+2}}\right] \cdot \frac{1}{\tau} I_{0}^{\frac{2 m+2-n}{n+2}} \geq \cdot \frac{1}{\tau \tau^{\prime}} I_{0}^{\frac{2 m+2}{n+2}} .
$$

Suppose we have defined $T_{0}=0, T_{1}, \cdots, T_{i}$ according to the above method and the following are tenable for $k=1,2, \cdots, i$ :

$$
\begin{gathered}
j_{k}-j_{k-1}=\left[\frac{1}{\tau^{\prime k}} I_{j_{k-1}}^{\frac{n}{n+2}}\right], \quad T_{k}-T_{k-1} \cdot \leq \frac{1}{\tau^{\prime k}}, \\
\sigma=\tau_{k}=\frac{1}{\tau^{k}}, \quad I_{j_{k}}:=I^{j_{k}}\left(T_{k}\right) \geq \cdot \frac{1}{\left(\tau \tau^{\prime}\right)^{k}} I_{0}^{\frac{2 m+2}{n+2}} .
\end{gathered}
$$

Set

$$
j_{i+1}-j_{i}=\left[\frac{1}{\tau^{\prime i+1}} I_{j_{i}}^{\frac{n}{n+2}}\right], \quad \sigma=\tau_{i+1}=\frac{1}{\tau^{i+1}}, \quad T_{i+1}:=t_{j_{i+1}},
$$

similar to the above discussion, we have

$$
I_{j_{i+1}}:=I^{j_{i+1}}\left(T_{i+1}\right) \geq \cdot \frac{1}{\left(\tau \tau^{\prime}\right)^{i+1}} I_{0}^{\frac{2 m+2}{n+2}}, \quad T_{i+1}-T_{i} \cdot \leq \frac{1}{\tau^{\prime i+1}} .
$$


Consequently,

$$
T_{i+1} \cdot \leq \sum_{k=1}^{i+1} \frac{1}{\tau^{\prime k}} \cdot \leq \frac{1}{\tau^{\prime}}<1
$$

if $\tau^{\prime}>0$ is sufficiently large.

Let

$$
\lim _{i \rightarrow \infty} T_{i}=T_{\infty}, \quad \lim _{i \rightarrow \infty} p^{i}(t)=p(t)
$$

since

$$
\max _{t_{1}, t_{2} \in\left[T_{k}, T_{\infty}\right]}\left|p\left(t_{1}\right)-p\left(t_{2}\right)\right| \leq \frac{1}{\tau^{k}}, \quad \lim _{t \rightarrow t_{\infty}} p(t)=1,
$$

then $p(t)$ can be extended to a continuous positive 1-periodic function.

Lemma 3.5. If $I_{0}$ is sufficiently large, then

$$
I_{j_{k}} \geq \cdot I_{0}^{k}
$$

where the constant $l>1$.

Proof. First, by the assumption $2 m+1 \geq n+2$, if we let $l=\frac{2 m+1}{n+2}+\frac{1}{2(n+2)}$, then $l>1$, and for sufficiently large $I_{0}$ we have

$$
I_{j_{1}} \geq \cdot \frac{1}{\tau \tau^{\prime}} I_{0}^{\frac{2 m+2}{n+2}} \geq \cdot \frac{1}{\tau \tau^{\prime}} I_{0}^{\frac{1}{2(n+2)}} I_{0}^{l} \geq \cdot I_{0}^{l} .
$$

If

$$
I_{j_{k}} \geq \cdot I_{0}^{l^{k}}
$$

then

$$
\begin{aligned}
I_{j_{k+1}} & \geq \cdot \frac{1}{\left(\tau \tau^{\prime}\right)^{k+1}} I_{j_{k}+2}^{\frac{2 m+2}{n+2}} \\
& \geq \cdot \frac{1}{\left(\tau \tau^{\prime}\right)^{k+1}} I_{0}^{\frac{2 m+2}{n+2} l^{k}} \\
& \geq \cdot \frac{I_{0}^{\frac{l^{k}}{2(n+2)}}}{\left(\tau \tau^{\prime}\right)^{k+1}} I_{0}^{l^{k+1}} \\
& \geq \cdot I_{0}^{k+1} .
\end{aligned}
$$

Proof of Theorem $1.1 \quad$ By Lemma 3.5, one has

$$
\min _{t \in\left[T_{i}, T_{i+1}\right]} I(t) \geq \cdot I_{j_{i}} \geq \cdot I_{0}^{l^{i}}
$$


Since $l>1$, then $I(t) \rightarrow+\infty$ as $t \rightarrow t_{\infty}$. Therefore, Eq.(1.3) in Theorem 1.1 possesses an unbounded solution defined in the interval $\left[0, T_{\infty}\right)$, and Theorem 1.1 is proved.

\section{Reference}

\section{References}

[1] R. Diekerhoff, E. Zehnder, Boundedness for solutions via the twist theorem, Ann. Scuola. Norm. Sup. Pisa. 14 (1987), 79-95.

[2] M. Levi, Quasiperiodic motions in superquadratic time periodic potentials, Commum. Math. Phys. 143 (1991), 43-83.

[3] M. Levi, On Littlewood's counterexample of unbounded motions in superquadratic potentials, Forschungsinstitut für Mathematik, ETH Zurich, 1992.

[4] M. Levi, J. You, Ocillatory escape in a Duffing equation with polynomial potential, J. Differential Equations 140 (1997), 415-426.

[5] X. Li, B. Liu, Y. Sun, The large twist theorem and boundedness of solutions for polynomial potentials with $C^{1}$ time dependent coefficients, Preprint.

[6] J. Littlewood, Unbounded solutions of $y^{\prime \prime}+g(y)=p(t)$, J. London Math. Soc. 41 (1966), 491-496.

[7] J. Littlewood, Unbounded solutions of an equation $y^{\prime \prime}+g(y)=p(t)$, with $p(t)$ periodic and bounded and $g(y) / y \rightarrow \infty$ as $y \rightarrow+\infty$, J. Lond. Math. Soc. 41 (1966), 497-507.

[8] J. Littlewood, Some Problems in Real and Complex Analysis, Heath, Lexington, Massachusetts, 1968.

[9] B. Liu, Boundedness for solutions of nonlinear Hill's equation with periodic forcing terms via Moser's twist theorem, J. Differential Equations, 79 (1989), 304-315.

[10] B. Liu, Boundedness for solutions of nonlinear periodic differential equations via Moser's twist theorem, Acta Mathematica Sinica N.S. 8 (1992), 91-98. 
[11] B. Liu, The stability of the equilibrium of a conservative system, J. Math. Anal. Appl. 202 (1996), 133-149.

[12] Y. Long, An unbounded solution of a superlinear Duffing's equation, Acta Mathematica Sinica 7 (1991), 360-369.

[13] S. Marò, Coexistence of bounded and unbounded motions in a bouncing ball model, Nonlinearity, 26 (2013), 1439-1448.

[14] G. Morris, A case of boundedness in Littlewood's problem on oscillatory differential equations, Bul. Austral. Math. Soc. 14 (1976), 71-93.

[15] J. Moser, On invariant curves of area-preserving mappings of an annulus, Nachr. Akad. Wiss. Gottingen Math. Phys. KI.II, (1962), 1-20.

[16] Y. Wang, Unboundedness in a Duffing equation with polynomial potentials, J. Differential Equations, 160 (2000), 467-479.

[17] Y. Wang, Boundedness of solutions in a class of Duffing equations with oscillating potentials, Nonlinear Anal. 71 (2009), 2906-2917.

[18] Z. Wang, Y. Wang, Boundedness of solutions for a class of oscillating potentials without the twist condition, Acta Mathematica Sinica 26 (2010), 2387-2398.

[19] Z. Wang, Y. Wang, H. Lu, The coexistence of quasi-periodic and blow-up solutions in a class of Hamiltonian systems, J. Math. Anal. Appl. 388 (2012), 888-898.

[20] X. Yuan, Invariant tori of Duffng-type equations, J. Differential Equations 142 (1998), no.2, 231-262. 